\title{
On The Dragomir Extension of Furuta's Inequality and Numerical Radius Inequalities
}

\author{
Mohammad W. Alomari \\ Department of Mathematics, Faculty of Science and Information Technology, Irbid National \\ University, 2600 Irbid 21110, Jordan; mwomath@gmail.com
}

\begin{abstract}
In this work, some numerical radius inequalities based on the re-cent Dragomir extension of Furuta's inequality are obtained. Some particular cases are also provided.
\end{abstract}

Keywords: mixed Schwarz inequality; Furuta inequality; numerical radius inequalities

\section{INTRODUCTION}

Let $\mathscr{B}(\mathscr{H})$ be the Banach algebra of all bounded linear operators defined on a complex Hilbert space $(\mathscr{H} ;\langle\cdot, \cdot\rangle)$ with the identity operator $1_{\mathscr{H}}$ in $\mathscr{B}(\mathscr{H})$.

The Schwarz inequality for positive operators reads that if $A$ is a positive operator in $\mathscr{B}(\mathscr{H})$, then

$$
|\langle A x, y\rangle|^{2} \leq\langle A x, x\rangle\langle A y, y\rangle
$$

for any vectors $x, y \in \mathscr{H}$.

In 1951, Reid [19] proved an inequality which in some senses considered a variant of the Schwarz inequality. In fact, he proved that for all operators $A \in$ $\mathscr{B}(\mathscr{H})$ such that $A$ is positive and $A B$ is selfadjoint then

$$
|\langle A B x, y\rangle| \leq\|B\|\langle A x, x\rangle,
$$

for all $x \in \mathscr{H}$. In [9], Halmos presented his stronger version of the Reid inequality (1.2) by replacing $r(B)$ instead of $\|B\|$.

In 1952, Kato [16] introduced a companion inequality of (1.1), called the mixed Schwarz inequality, which asserts

$$
|\langle A x, y\rangle|^{2} \leq\left\langle|A|^{2 \alpha} x, x\right\rangle\left\langle\left|A^{*}\right|^{2(1-\alpha)} y, y\right\rangle, \quad 0 \leq \alpha \leq 1 .
$$

for every operators $A \in \mathscr{B}(\mathscr{H})$ and any vectors $x, y \in \mathscr{H}$, where $|A|=\left(A^{*} A\right)^{1 / 2}$.

In 1988, Kittaneh [14] proved a very interesting extension combining both the Halmos-Reid inequality (1.2) and the mixed Schwarz inequality (1.3). His result reads that

$$
|\langle A B x, y\rangle| \leq r(B)\|f(|A|) x\|\left\|g\left(\left|A^{*}\right|\right) y\right\|
$$

for any vectors $x, y \in \mathscr{H}$, where $A, B \in \mathscr{B}(\mathscr{H})$ such that $|A| B=B^{*}|A|$ and $f, g$ are nonnegative continuous functions defined on $[0, \infty)$ satisfying that $f(t) g(t)=t$ $(t \geq 0)$. Clearly, choose $f(t)=t^{\alpha}$ and $g(t)=t^{1-\alpha}$ with $B=1_{\mathscr{H}}$ we refer to (1.3). Moreover, choosing $\alpha=\frac{1}{2}$ some manipulations refer to the Halmos version of the

Date: December 5, 2019.

2010 Mathematics Subject Classification. Primary: 47A30, 47A12 Secondary: 15A60, 47A63. Key words and phrases. Mixed Schwarz inequality, Furuta inequality, Numerical radius inequalities. 
Reid inequality. The cartesian decomposition form of (1.4) was recently proved by the Alomari in [2].

In 1994, Furuta [8] proved the another attractive generalization of Kato's inequality (1.3), as follows:

$$
\left|\left\langle T|T|^{\alpha+\beta-1} x, y\right\rangle\right|^{2} \leq\left\langle|T|^{2 \alpha} x, x\right\rangle\left\langle|T|^{2 \beta} y, y\right\rangle
$$

for any $x, y \in \mathscr{H}$ and $\alpha, \beta \in[0,1]$ with $\alpha+\beta \geq 1$.

The inequality (1.5) was generalized for any $\alpha, \beta \geq 0$ with $\alpha+\beta \geq 1$ by Dragomir in [7]. Indeed, as noted by Dragomir the condition $\alpha, \beta \in[0,1]$ was assumed by Furuta to fit with the Heinz-Kato inequality, which reads:

$$
|\langle T x, y\rangle| \leq\left\|A^{\alpha} x\right\|\left\|B^{1-\alpha} y\right\|
$$

for any $x, y \in \mathscr{H}$ and $\alpha \in[0,1]$ where $A$ and $B$ are positive operators such that $\|T x\| \leq\|A x\|$ and $\left\|T^{*} y\right\| \leq\|B y\|$ for any $x, y \in \mathscr{H}$.

In the same work [7], Dragomir provides a useful extension of Furuta's inequality, as follows:

$$
|\langle D C B A x, y\rangle|^{2} \leq\left\langle A^{*}|B|^{2} A x, x\right\rangle\left\langle D\left|C^{*}\right|^{2} D^{*} y, y\right\rangle
$$

for any $A, B, C, D \in \mathscr{B}(\mathscr{H})$ and any vectors $x, y \in \mathscr{H}$. The equality in (1.6) holds iff the vectors $B A x$ and $C^{*} D^{*} y$ are linearly dependent in $\mathscr{H}$.

Indeed, since $A^{*}|B|^{2} A=A^{*} B^{*} B A=\left(A^{*} B^{*}\right)(B A)=(B A)^{*}(B A)=|B A|^{2}$ and $D\left|C^{*}\right|^{2} D^{*}=D C C^{*} D^{*}=(D C)\left(C^{*} D^{*}\right)=(D C)(D C)^{*}=\left|(D C)^{*}\right|^{2}=\left|C^{*} D^{*}\right|^{2}$. Therefore, (1.6) can be rewritten as:

$$
|\langle D C B A x, y\rangle|^{2} \leq\left\langle|B A|^{2} x, x\right\rangle\left\langle\left|C^{*} D^{*}\right|^{2} y, y\right\rangle .
$$

If one setting $D=U$ ( $U$ is unitary), $B=1_{\mathscr{H}}, C=|T|^{\beta}$ and $A=|T|^{\alpha}$ such that $\alpha+\beta \geq 1$, then we recapture (1.5).

For a bounded linear operator $T$ on a Hilbert space $\mathscr{H}$, the numerical range $W(T)$ is the image of the unit sphere of $\mathscr{H}$ under the quadratic form $x \rightarrow\langle T x, x\rangle$ associated with the operator. More precisely,

$$
W(T)=\{\langle T x, x\rangle: x \in \mathscr{H},\|x\|=1\}
$$

Also, the numerical radius is defined to be

$$
w(T)=\sup \{|\lambda|: \lambda \in W(T)\}=\sup _{\|x\|=1}|\langle T x, x\rangle| .
$$

The spectral radius of an operator $T$ is defined to be

$$
r(T)=\sup \{|\lambda|: \lambda \in \operatorname{sp}(T)\} .
$$

We recall that, the usual operator norm of an operator $T$ is defined to be

$$
\|T\|=\sup \{\|T x\|: x \in H,\|x\|=1\} .
$$

It is well known that $w(\cdot)$ defines an operator norm on $\mathscr{B}(\mathscr{H})$ which is equivalent to operator norm $\|\cdot\|$. Moreover, we have

$$
\frac{1}{2}\|T\| \leq w(T) \leq\|T\|
$$

for any $T \in \mathscr{B}(\mathscr{H})$ and this inequality is sharp. 
In 2003, Kittaneh [14] refined the right-hand side of (1.8), where he proved that

$$
w(T) \leq \frac{1}{2}\left(\|T\|+\left\|T^{2}\right\|^{1 / 2}\right)
$$

for any $T \in \mathscr{B}(\mathscr{H})$.

After that in 2005, the same author in [12] proved that

$$
\frac{1}{4}\left\|A^{*} A+A A^{*}\right\| \leq w^{2}(A) \leq \frac{1}{2}\left\|A^{*} A+A A^{*}\right\| .
$$

The inequality is sharp.

In 2007, Yamazaki [22] improved (1.8) by proving that

$$
w(T) \leq \frac{1}{2}(\|T\|+w(\widetilde{T})) \leq \frac{1}{2}\left(\|T\|+\left\|T^{2}\right\|^{1 / 2}\right)
$$

where $\widetilde{T}=|T|^{1 / 2} U|T|^{1 / 2}$ and $U$ is the unitary operator in the polar decomposition $T$ of the form $T=U|T|$.

In 2008, Dragomir [6] used Buzano inequality to improve (1.1), where he proved that

$$
w^{2}(T) \leq \frac{1}{2}\left(\|T\|+w\left(T^{2}\right)\right)
$$

This result was also recently generalized by Sattari et al. in [20] and Alomari in [3]. For more recent results about the numerical radius see the recent monograph study [5].

In this work, some numerical radius inequalities based on the recent Dragomir extension of Furuta's inequality are obtained. Some particular cases are also provided.

\section{LEMMAS}

2.1. Preliminaries. In order to prove our main result we need to the following Lemmas:

Lemma 2.1. Let $S \in \mathscr{B}(\mathscr{H}), S \geq 0$ and $x \in \mathscr{H}$ be a unit vector. Then, the operator Jensen's inequality

$$
\langle S x, x\rangle^{r} \leq(\geq)\left\langle S^{r} x, x\right\rangle, \quad r \geq 1 \quad(0 \leq r \leq 1) .
$$

Kittaneh and Manasrah [13] obtained the following result which is a refinement of the scalar Young inequality.

Lemma 2.2. Let $a, b \geq 0$, and $p, q>1$ such that $\frac{1}{p}+\frac{1}{q}=1$. Then

$$
a b+\min \left\{\frac{1}{p}, \frac{1}{q}\right\}\left(a^{\frac{p}{2}}-b^{\frac{q}{2}}\right)^{2} \leq \frac{a^{p}}{p}+\frac{b^{q}}{q} .
$$

Manasrah and Kittaneh have generalized (2.3) in [1], as follows:

Lemma 2.3. If $a, b>0$, and $p, q>1$ such that $\frac{1}{p}+\frac{1}{q}=1$, then for $m=1,2,3, \ldots$,

$$
\left(a^{\frac{1}{p}} b^{\frac{1}{q}}\right)^{m}+r_{0}^{m}\left(a^{\frac{m}{2}}-b^{\frac{m}{2}}\right)^{2} \leq\left(\frac{a^{r}}{p}+\frac{b^{r}}{q}\right)^{\frac{m}{r}}, r \geq 1
$$


where $r_{0}=\min \left\{\frac{1}{p}, \frac{1}{q}\right\}$. In particular, if $p=q=2$, then

$$
\left(a^{\frac{1}{2}} b^{\frac{1}{2}}\right)^{m}+\frac{1}{2^{m}}\left(a^{\frac{m}{2}}-b^{\frac{m}{2}}\right)^{2} \leq 2^{\frac{-m}{r}}\left(a^{r}+b^{r}\right)^{\frac{m}{r}} .
$$

For $m=1$

$$
\left(a^{\frac{1}{2}} b^{\frac{1}{2}}\right)+\frac{1}{2}\left(a^{\frac{1}{2}}-b^{\frac{1}{2}}\right)^{2} \leq 2^{\frac{-1}{r}}\left(a^{r}+b^{r}\right)^{\frac{1}{r}} .
$$

Lemma 2.4. ([10]) Let $f$ be a twice differentiable on $[a, b]$. If $f$ is convex such that $f^{\prime \prime} \geq \lambda:=\min _{x \in[a, b]} f(x)>0$. Then

$$
f\left(\frac{a+b}{2}\right) \leq \frac{f(a)+f(b)}{2}-\frac{1}{8} \lambda(b-a)^{2} .
$$

Lemma 2.5. ([18]) Let $f$ be a convex function defined on a real interval $I$. Then for every selfadjoint operator $A \in \mathscr{B}(\mathscr{H})$ whose $\operatorname{sp}(A) \subset I$, we have

$$
f(\langle A x, x\rangle) \leq\langle f(A) x, x\rangle
$$

for all vector $x \in \mathscr{H}$

2.2. Extensions of the Dragomir-Furuta inequality. In this section we provide some key lemmas which are plays the main role in the proof of our main results.

Lemma 2.6. Let $A, B, C, D \in \mathscr{B}(\mathscr{H})$. Let $f$ be a positive, increasing and convex functions on $\mathbb{R}$. If $f$ is twice differentiable such that $f^{\prime \prime} \geq \lambda>0$, then

$$
\begin{aligned}
f(|\langle D C B A x, y\rangle|) \leq \frac{1}{2}\left[\left\langle f\left(A^{*}|B|^{2} A\right) x, x\right\rangle+\left\langle f\left(D\left|C^{*}\right|^{2} D^{*}\right) y, y\right\rangle\right] \\
-\frac{1}{8} \lambda\left(\left\langle A^{*}|B|^{2} A x, x\right\rangle-\left\langle D\left|C^{*}\right|^{2} D^{*} y, y\right\rangle\right)^{2}
\end{aligned}
$$

for all vectors $x, y \in \mathscr{H}$.

Proof. Employing the monotonicity and convexity of $f$ for the inequality (1.6), we have

$$
\begin{aligned}
& f(|\langle D C B A x, y\rangle|) \leq f\left(\left\langle A^{*}|B|^{2} A x, x\right\rangle^{\frac{1}{2}}\left\langle D\left|C^{*}\right|^{2} D^{*} y, y\right\rangle^{\frac{1}{2}}\right) \quad \text { (f increasing) } \\
& \leq f\left(\frac{\left\langle A^{*}|B|^{2} A x, x\right\rangle+\left\langle D\left|C^{*}\right|^{2} D^{*} y, y\right\rangle}{2}\right) \quad \text { (by AM-GM inequality) } \\
& \leq \frac{f\left(\left\langle A^{*}|B|^{2} A x, x\right\rangle\right)+f\left(\left\langle D\left|C^{*}\right|^{2} D^{*} y, y\right\rangle\right)}{2} \quad \text { (by Lemma 2.4) } \\
& -\frac{1}{8} \lambda\left(\left\langle A^{*}|B|^{2} A x, x\right\rangle-\left\langle D\left|C^{*}\right|^{2} D^{*} y, y\right\rangle\right)^{2} \\
& \leq \frac{1}{2}\left[\left\langle f\left(A^{*}|B|^{2} A\right) x, x\right\rangle+\left\langle f\left(D\left|C^{*}\right|^{2} D^{*}\right) y, y\right\rangle\right] \quad \text { (by Lemma 2.5) } \\
& -\frac{1}{8} \lambda\left(\left\langle A^{*}|B|^{2} A x, x\right\rangle-\left\langle D\left|C^{*}\right|^{2} D^{*} y, y\right\rangle\right)^{2}
\end{aligned}
$$

for all vectors $x, y \in \mathscr{H}$, which proves the result. 
Corollary 2.7. Let $T \in \mathscr{B}(\mathscr{H})$. Let $f$ be a positive, increasing and convex functions on $\mathbb{R}$. If $f$ is twice differentiable such that $f^{\prime \prime} \geq \lambda>0$, then

$$
\begin{array}{r}
f\left(\left|\left\langle T|T|^{\alpha+\beta-1} x, y\right\rangle\right|\right) \leq \frac{1}{2}\left[\left\langle f\left(|T|^{2 \alpha}\right) x, x\right\rangle+\left\langle f\left(\left|T^{*}\right|^{2 \beta}\right) y, y\right\rangle\right] \\
-\frac{1}{8} \lambda\left(\left\langle|T|^{2 \alpha} x, x\right\rangle-\left\langle\left|T^{*}\right|^{2 \beta} y, y\right\rangle\right)^{2}
\end{array}
$$

for all vectors $x, y \in \mathscr{H}$ and all $\alpha, \beta \in[0,1]$ such that $\alpha+\beta \geq 1$.

Proof. Let $D=U, B=1_{\mathscr{H}}, C=|T|^{\beta}$ and $A=|T|^{\alpha}$ such that $\alpha+\beta \geq 1$ in (2.5), then we have

$$
D C B A=U|T|^{\beta}|T|^{\alpha}=U|T||T|^{\alpha+\beta-1}=T|T|^{\alpha+\beta-1},
$$

also, we have $A^{*}|B|^{2} A=|T|^{2 \alpha}$ and $D\left|C^{*}\right|^{2} D^{*}=U|T|^{2 \beta} U^{*}=|T|^{2 \beta}$, and this proves the required result.

Lemma 2.8. Let $f: \mathbb{R} \rightarrow(0, \infty)$ be a positive, increasing, convex and supermultiplicative i.e., $f(t s) \leq f(t) f(s)$ for all $t, s \in \mathbb{R}$.

$$
\begin{aligned}
f\left(|\langle D C B A x, y\rangle|^{2}\right) \leq & \frac{1}{p}\left\langle f^{p}\left(A^{*}|B|^{2} A\right) x, x\right\rangle+\frac{1}{q}\left\langle f^{q}\left(D\left|C^{*}\right|^{2} D^{*}\right) y, y\right\rangle \\
& -r_{0}\left(\left\langle f\left(A^{*}|B|^{2} A\right) x, x\right\rangle^{\frac{p}{2}}-\left\langle f\left(D\left|C^{*}\right|^{2} D^{*}\right) y, y\right\rangle^{\frac{q}{2}}\right)^{2}
\end{aligned}
$$

for all vectors $x, y \in \mathscr{H}$.

Proof. From (1.6) we have

$$
\begin{aligned}
& f\left(|\langle D C B A x, y\rangle|^{2}\right) \\
& \leq f\left(\left\langle A^{*}|B|^{2} A x, x\right\rangle\left\langle D\left|C^{*}\right|^{2} D^{*} y, y\right\rangle\right) \quad(f \text { increasing }) \\
& \leq f\left(\left\langle A^{*}|B|^{2} A x, x\right\rangle\right) f\left(\left\langle D\left|C^{*}\right|^{2} D^{*} y, y\right\rangle\right) \quad \text { (f supermultiplicative) } \\
& \leq\left\langle f\left(A^{*}|B|^{2} A\right) x, x\right\rangle\left\langle f\left(D\left|C^{*}\right|^{2} D^{*}\right) y, y\right\rangle \quad \text { (by Lemma 2.5) } \\
& \leq \frac{1}{p}\left\langle f\left(A^{*}|B|^{2} A\right) x, x\right\rangle^{p}+\frac{1}{q}\left\langle f\left(D\left|C^{*}\right|^{2} D^{*}\right) y, y\right\rangle^{q} \quad \text { (by Lemma 2.2) } \\
& \quad-r_{0}\left(\left\langle f\left(A^{*}|B|^{2} A\right) x, x\right\rangle^{\frac{p}{2}}-\left\langle f\left(D\left|C^{*}\right|^{2} D^{*}\right) y, y\right\rangle^{\frac{q}{2}}\right)^{2} \\
& \leq \frac{1}{p}\left\langle f^{p}\left(A^{*}|B|^{2} A\right) x, x\right\rangle+\frac{1}{q}\left\langle f^{q}\left(D\left|C^{*}\right|^{2} D^{*}\right) y, y\right\rangle \\
& \quad-r_{0}\left(\left\langle f\left(A^{*}|B|^{2} A\right) x, x\right\rangle^{\frac{p}{2}}-\left\langle f\left(D\left|C^{*}\right|^{2} D^{*}\right) y, y\right\rangle^{\frac{q}{2}}\right)^{2}
\end{aligned}
$$

for all vectors $x, y \in \mathscr{H}$.

Corollary 2.9. Let $f: \mathbb{R} \rightarrow(0, \infty)$ be a positive, increasing, convex and supermultiplicative i.e., $f(t s) \leq f(t) f(s)$ for all $t, s \in \mathbb{R}$.

$$
\begin{aligned}
f\left(\left|\left\langle T|T|^{\alpha+\beta-1} x, y\right\rangle\right|^{2}\right) \leq & \frac{1}{p}\left\langle f^{p}\left(|T|^{2 \alpha}\right) x, x\right\rangle+\frac{1}{q}\left\langle f^{q}\left(\left|T^{*}\right|^{2 \beta}\right) y, y\right\rangle \\
& -r_{0}\left(\left\langle f\left(|T|^{2 \alpha}\right) x, x\right\rangle^{\frac{p}{2}}-\left\langle f\left(\left|T^{*}\right|^{2 \alpha}\right) y, y\right\rangle^{\frac{q}{2}}\right)^{2}
\end{aligned}
$$


for all vectors $x, y \in \mathscr{H}$.

Proof. The proof goes likewise the proof of Corollary 2.7 taking into account Lemma 2.8.

Lemma 2.10. Let $f: \mathbb{R} \rightarrow(0, \infty)$ be a positive, increasing, convex and supermultiplicative i.e., $f(t s) \leq f(t) f(s)$ for all $t, s \in \mathbb{R}$.

$$
\begin{aligned}
f\left(|\langle D C B A x, y\rangle|^{2}\right) \leq 2^{-\frac{2}{r}} & \left(\left\langle f^{r}\left(A^{*}|B|^{2} A\right) x, x\right\rangle+\left\langle f^{r}\left(D\left|C^{*}\right|^{2} D^{*}\right) y, y\right\rangle\right)^{\frac{2}{r}} \\
& -\frac{1}{4}\left[\left\langle f\left(A^{*}|B|^{2} A\right) x, x\right\rangle-\left\langle f\left(D\left|C^{*}\right|^{2} D^{*}\right) y, y\right\rangle\right]
\end{aligned}
$$

for all $r \geq 1$. In particular case, we have

$$
\begin{aligned}
f\left(|\langle D C B A x, y\rangle|^{2}\right) \leq & \frac{1}{4}\left(\left\langle f\left(A^{*}|B|^{2} A\right) x, x\right\rangle+\left\langle f\left(D\left|C^{*}\right|^{2} D^{*}\right) y, y\right\rangle\right)^{2} \\
& -\frac{1}{4}\left[\left\langle f\left(A^{*}|B|^{2} A\right) x, x\right\rangle-\left\langle f\left(D\left|C^{*}\right|^{2} D^{*}\right) y, y\right\rangle\right]
\end{aligned}
$$

for all vectors $x, y \in \mathscr{H}$.

Proof. Since $f$ is increasing and convex, then by applying Lemma 2.3, with $p=$ $q=2$ and $m=2$, we get

$f\left(|\langle D C B A x, y\rangle|^{2}\right)$

$$
\begin{aligned}
& \leq f\left(\left\langle A^{*}|B|^{2} A x, x\right\rangle\left\langle D\left|C^{*}\right|^{2} D^{*} y, y\right\rangle\right) \quad \text { (f increasing) } \\
& \leq f\left(\left\langle A^{*}|B|^{2} A x, x\right\rangle\right) f\left(\left\langle D\left|C^{*}\right|^{2} D^{*} y, y\right\rangle\right) \quad \text { (f supermultiplicative) } \\
& \leq\left\langle f\left(A^{*}|B|^{2} A\right) x, x\right\rangle\left\langle f\left(D\left|C^{*}\right|^{2} D^{*}\right) y, y\right\rangle \quad \text { (by Lemma 2.5) } \\
& \leq 2^{-\frac{2}{r}}\left(\left\langle f\left(A^{*}|B|^{2} A\right) x, x\right\rangle^{r}+\left\langle f\left(D\left|C^{*}\right|^{2} D^{*}\right) y, y\right\rangle^{r}\right)^{\frac{2}{r}} \quad \text { (by Lemma 2.3) } \\
& -\frac{1}{4}\left[\left\langle f\left(A^{*}|B|^{2} A\right) x, x\right\rangle-\left\langle f\left(D\left|C^{*}\right|^{2} D^{*}\right) y, y\right\rangle\right] \\
& \leq 2^{-\frac{2}{r}}\left(\left\langle f^{r}\left(A^{*}|B|^{2} A\right) x, x\right\rangle+\left\langle f^{r}\left(D\left|C^{*}\right|^{2} D^{*}\right) y, y\right\rangle\right)^{\frac{2}{r}} \quad \text { (by Lemma 2.1) } \\
& -\frac{1}{4}\left[\left\langle f\left(A^{*}|B|^{2} A\right) x, x\right\rangle-\left\langle f\left(D\left|C^{*}\right|^{2} D^{*}\right) y, y\right\rangle\right]
\end{aligned}
$$

for all vectors $x, y \in \mathscr{H}$.

Corollary 2.11. Let $f: \mathbb{R} \rightarrow(0, \infty)$ be a positive, increasing, convex and supermultiplicative i.e., $f(t s) \leq f(t) f(s)$ for all $t, s \in \mathbb{R}$.

$$
\begin{aligned}
f\left(\left|\left\langle T|T|^{\alpha+\beta-1} x, y\right\rangle\right|^{2}\right) \leq & 2^{-\frac{2}{r}} \\
& \left(\left\langle f^{r}\left(|T|^{2 \alpha}\right) x, x\right\rangle+\left\langle f^{r}\left(\left|T^{*}\right|^{2 \beta}\right) y, y\right\rangle\right)^{\frac{2}{r}} \\
& -\frac{1}{4}\left[\left\langle f\left(|T|^{2 \alpha}\right) x, x\right\rangle-\left\langle f\left(\left|T^{*}\right|^{2 \beta}\right) y, y\right\rangle\right]
\end{aligned}
$$


In particular case, we have

$$
\begin{aligned}
f\left(\left|\left\langle T|T|^{\alpha+\beta-1} x, y\right\rangle\right|^{2}\right) \leq & \frac{1}{4} \\
& \left(\left\langle f\left(|T|^{2 \alpha}\right) x, x\right\rangle+\left\langle\left(\left|T^{*}\right|^{2 \beta}\right) y, y\right\rangle\right)^{2} \\
& -\frac{1}{4}\left[\left\langle f\left(|T|^{2 \alpha}\right) x, x\right\rangle-\left\langle f\left(\left|T^{*}\right|^{2 \beta}\right) y, y\right\rangle\right]
\end{aligned}
$$

for all vectors $x, y \in \mathscr{H}$.

Proof. The proof of (2.7) goes likewise the proof of Corollary 2.7 taking into account Lemma 2.10.

\section{Numerical Radius inequalities}

In this section we provide some numerical radius inequalities. Let us begin with the following result.

Theorem 3.1. Let $A, B, C, D \in \mathscr{B}(\mathscr{H})$. Let $f$ be a positive, increasing and convex functions on $\mathbb{R}$. If $f$ is twice differentiable such that $f^{\prime \prime} \geq \lambda>0$, then

$$
f(w(D C B A)) \leq \frac{1}{2}\left\|f\left(A^{*}|B|^{2} A\right)+f\left(D\left|C^{*}\right|^{2} D^{*}\right)\right\|-\inf _{\|x\|=1} \eta(x),
$$

where $\eta(x):=\frac{1}{8} \lambda\left\langle\left[A^{*}|B|^{2} A-D\left|C^{*}\right|^{2} D^{*}\right] x, x\right\rangle^{2}$

Proof. Let $y=x$ in (2.5), then we get

$$
\begin{aligned}
f(|\langle D C B A x, x\rangle|) \leq \frac{1}{2}[ & \left.\left\langle f\left(A^{*}|B|^{2} A\right) x, x\right\rangle+\left\langle f\left(D\left|C^{*}\right|^{2} D^{*}\right) x, x\right\rangle\right] \\
& -\frac{1}{8} \lambda\left(\left\langle A^{*}|B|^{2} A x, x\right\rangle-\left\langle D\left|C^{*}\right|^{2} D^{*} x, x\right\rangle\right)^{2} \\
= & \frac{1}{2}\left\langle\left[f\left(A^{*}|B|^{2} A\right)+f\left(D\left|C^{*}\right|^{2} D^{*}\right)\right] x, x\right\rangle \\
& -\frac{1}{8} \lambda\left\langle\left[A^{*}|B|^{2} A-D\left|C^{*}\right|^{2} D^{*}\right] x, x\right\rangle^{2} .
\end{aligned}
$$

Taking the supremum over all univt vector $x \in \mathscr{H}$, we get the required result.

Corollary 3.2. Let $A, B, C, D \in \mathscr{B}(\mathscr{H})$. Then

$$
\begin{aligned}
w^{2}(D C B A) \leq \frac{1}{2} \|\left(A^{*}|B|^{2} A\right)^{2}+ & \left(D\left|C^{*}\right|^{2} D^{*}\right)^{2} \| \\
& -\inf _{\|x\|=1} \frac{1}{4}\left\langle\left[A^{*}|B|^{2} A-D\left|C^{*}\right|^{2} D^{*}\right] x, x\right\rangle^{2}
\end{aligned}
$$

Proof. Take $f(x)=x^{2}$ in Theorem 3.1, thus the required $\lambda$ would be '2'.

Corollary 3.3. Let $T \in \mathscr{B}(\mathscr{H})$. Let $f$ be a positive, increasing and convex functions on $\mathbb{R}$. If $f$ is twice differentiable such that $f^{\prime \prime} \geq \lambda>0$, then

$$
f\left(w\left(T|T|^{\alpha+\beta-1}\right)\right) \leq \frac{1}{2}\left\|f\left(|T|^{2 \alpha}\right)+f\left(\left|T^{*}\right|^{2 \beta}\right)\right\|-\inf _{\|x\|=1} \xi(x)
$$

where $\xi(x):=\frac{1}{8} \lambda\left\langle\left[|T|^{2 \alpha}-\left|T^{*}\right|^{2 \beta}\right] x, x\right\rangle^{2}$, for all such that $\alpha, \beta \in[0,1]$ such that $\alpha+\beta \geq 1$. 
Proof. Let $y=x$ in (2.6), we get

$$
\begin{aligned}
f\left(\left|\left\langle T|T|^{\alpha+\beta-1} x, x\right\rangle\right|\right) \leq \frac{1}{2}[ & \left.\left\langle f\left(|T|^{2 \alpha}\right) x, x\right\rangle+\left\langle f\left(\left|T^{*}\right|^{2 \beta}\right) x, x\right\rangle\right] \\
& -\frac{1}{8} \lambda\left(\left\langle|T|^{2 \alpha} x, x\right\rangle-\left\langle\left|T^{*}\right|^{2 \beta} x, x\right\rangle\right)^{2} .
\end{aligned}
$$

Taking the supremum over all univt vector $x \in \mathscr{H}$, we get the required result.

Corollary 3.4. Let $A, B \in \mathscr{B}(\mathscr{H})$. Let $f$ be a positive, increasing and convex functions on $\mathbb{R}$. If $f$ is twice differentiable such that $f^{\prime \prime} \geq \lambda>0$, then

$$
f\left(w\left((B A)^{2}\right)\right) \leq \frac{1}{2}\left\|f\left(A^{*}|B|^{2} A\right)+f\left(B\left|A^{*}\right|^{2} B^{*}\right)\right\|-\inf _{\|x\|=1} \eta_{1}(x),
$$

where $\eta_{1}(x):=\frac{1}{8} \lambda\left\langle\left[A^{*}|B|^{2} A-B\left|A^{*}\right|^{2} B^{*}\right] x, x\right\rangle^{2}$

Proof. Setting $D=B$ and $C=A$ in (3.1).

Corollary 3.5. Let $A, B \in \mathscr{B}(\mathscr{H})$. Let $f$ be a positive, increasing and convex functions on $\mathbb{R}$. If $f$ is twice differentiable such that $f^{\prime \prime} \geq \lambda>0$, then

$$
f\left(w\left(A^{*} B^{2} A\right)\right) \leq \frac{1}{2}\left\|f\left(A^{*}|B|^{2} A\right)+f\left(A^{*}\left|B^{*}\right|^{2} A\right)\right\|-\inf _{\|x\|=1} \eta_{2}(x),
$$

where $\eta_{2}(x):=\frac{1}{8} \lambda\left\langle\left[A^{*}|B|^{2} A-A^{*}\left|B^{*}\right|^{2} A\right] x, x\right\rangle^{2}$

Proof. Setting $D=A$ and $C=B$ in (3.1).

Corollary 3.6. Let $A \in \mathscr{B}(\mathscr{H})$. Let $f$ be a positive, increasing and convex functions on $\mathbb{R}$. If $f$ is twice differentiable such that $f^{\prime \prime} \geq \lambda>0$, then

$$
f\left(w\left(A^{4}\right)\right) \leq \frac{1}{2}\left\|f\left(A^{*}|A|^{2} A\right)+f\left(A\left|A^{*}\right|^{2} A^{*}\right)\right\|-\inf _{\|x\|=1} \eta(x),
$$

where $\eta(x):=\frac{1}{8} \lambda\left\langle\left[A^{*}|A|^{2} A-A\left|A^{*}\right|^{2} A^{*}\right] x, x\right\rangle^{2}$

Proof. Setting $D=C=B=A$ in (3.1).

Theorem 3.7. Let $A, B, C, D \in \mathscr{B}(\mathscr{H})$. Let $f: \mathbb{R} \rightarrow(0, \infty)$ be an increasing, convex and supermultiplicative i.e., $f(t s) \leq f(t) f(s)$ for all $t, s \in \mathbb{R}$.

$$
f\left(w^{2}(D C B A)\right) \leq\left\|\frac{1}{p} f^{p}\left(A^{*}|B|^{2} A\right)+\frac{1}{q} f^{q}\left(D\left|C^{*}\right|^{2} D^{*}\right)\right\|-\inf _{\|x\|=1} \psi(x)
$$

for all $\alpha, \beta \in[0,1]$ such that $\alpha+\beta \geq 1$ and all $p, q>1$ such that $\frac{1}{p}+\frac{1}{q}=1$, where

$$
\psi(x):=r_{0}\left(\left\langle f\left(A^{*}|B|^{2} A\right) x, x\right\rangle^{\frac{p}{2}}-\left\langle f\left(D\left|C^{*}\right|^{2} D^{*}\right) x, x\right\rangle^{\frac{q}{2}}\right)^{2} .
$$

Proof. Let $y=x$ in (2.7), we get

$$
\begin{aligned}
f\left(|\langle D C B A x, x\rangle|^{2}\right) \leq\langle & \left.\left\langle\frac{1}{p} f^{p}\left(A^{*}|B|^{2} A\right)+\frac{1}{q} f^{q}\left(D\left|C^{*}\right|^{2} D^{*}\right)\right] x, x\right\rangle \\
& -r_{0}\left(\left\langle f\left(A^{*}|B|^{2} A\right) x, x\right\rangle^{\frac{p}{2}}-\left\langle f\left(D\left|C^{*}\right|^{2} D^{*}\right) x, x\right\rangle^{\frac{q}{2}}\right)^{2}
\end{aligned}
$$

Taking the supremum over all univt vector $x \in \mathscr{H}$, we get the required result. 
Corollary 3.8. Let $T \in \mathscr{B}(\mathscr{H})$. Let $f: \mathbb{R} \rightarrow(0, \infty)$ be an increasing, convex and supermultiplicative i.e., $f(t s) \leq f(t) f(s)$ for all $t, s \in \mathbb{R}$. Then,

$$
f\left(w^{2}\left(T|T|^{\alpha+\beta-1}\right)\right) \leq\left\|\frac{1}{p} f^{p}\left(|T|^{2 \alpha}\right)+\frac{1}{q} f^{q}\left(\left|T^{*}\right|^{2 \beta}\right)\right\|-\inf _{\|x\|=1} \psi_{1}(x)
$$

for all $\alpha, \beta \in[0,1]$ such that $\alpha+\beta \geq 1$ and all $p, q>1$ such that $\frac{1}{p}+\frac{1}{q}=1$, where

$$
\psi_{1}(x):=r_{0}\left(\left\langle f\left(|T|^{2 \alpha}\right) x, x\right\rangle^{\frac{p}{2}}-\left\langle f\left(\left|T^{*}\right|^{2 \beta}\right) x, x\right\rangle^{\frac{q}{2}}\right)^{2}
$$

Proof. Let $y=x$ in (2.8), and then taking the supremum over all univt vector $x \in \mathscr{H}$, we get the required result.

Corollary 3.9. Let $T \in \mathscr{B}(\mathscr{H})$. Then,

$$
w^{2 r}\left(T|T|^{\alpha+\beta-1}\right) \leq\left\|\frac{1}{p}|T|^{2 r p \alpha}+\frac{1}{q}\left|T^{*}\right|^{2 r q \beta}\right\|-\inf _{\|x\|=1} \psi_{1}(x)
$$

for all $\alpha, \beta \in[0,1]$ such that $\alpha+\beta \geq 1$, where

$$
\psi_{1}(x):=r_{0}\left(\left\langle|T|^{2 r \alpha} x, x\right\rangle^{\frac{p}{2}}-\left\langle\left|T^{*}\right|^{2 r \beta} x, x\right\rangle^{\frac{q}{2}}\right)^{2}
$$

for all $p, q>1$ such that $\frac{1}{p}+\frac{1}{q}=1$.

Proof. Applying Corollary 3.8 for the convex increasing function $f(t)=t^{r},(t>$ 0) $r \geq 1$.

Remark 3.10. In (3.5), let $p=q=2$ we get

$$
w^{2 r}\left(T|T|^{\alpha+\beta-1}\right) \leq \frac{1}{2}\left\||T|^{4 r \alpha}+\left|T^{*}\right|^{4 r \beta}\right\|-\inf _{\|x\|=1} \psi_{2}(x)
$$

for all $\alpha, \beta \in[0,1]$ such that $\alpha+\beta \geq 1$, where

$$
\psi_{2}(x):=\frac{1}{2}\left(\left\langle|T|^{2 r \alpha} x, x\right\rangle-\left\langle\left|T^{*}\right|^{2 r \beta} x, x\right\rangle\right)^{2} .
$$

In particular, for $\alpha=\beta=\frac{1}{2}$ we have

$$
w^{2 r}(T) \leq \frac{1}{2}\left\||T|^{2 r}+\left|T^{*}\right|^{2 r}\right\|-\frac{1}{2} \inf _{\|x\|=1}\left(\left\langle|T|^{r} x, x\right\rangle-\left\langle\left|T^{*}\right|^{r} x, x\right\rangle\right)^{2} .
$$

for all $r \geq 1$.

Theorem 3.11. Let $A, B, C, D \in \mathscr{B}(\mathscr{H})$. Let $f: \mathbb{R} \rightarrow(0, \infty)$ be an increasing, convex and supermultiplicative i.e., $f(t s) \leq f(t) f(s)$ for all $t, s \in \mathbb{R}$.

$$
f\left(w^{2}(D C B A)\right) \leq 2^{-\frac{2}{r}}\left\|f^{r}\left(A^{*}|B|^{2} A\right)+f^{r}\left(D\left|C^{*}\right|^{2} D^{*}\right)\right\|^{\frac{2}{r}}-\inf _{\|x\|=1} \phi(x)
$$

where,

$$
\phi(x):=\frac{1}{4}\left[\left\langle\left[f\left(A^{*}|B|^{2} A\right)-f\left(D\left|C^{*}\right|^{2} D^{*}\right)\right] x, x\right\rangle\right]
$$


In particular case, we have

$$
f\left(w^{2}(D C B A)\right) \leq \frac{1}{4}\left\|f\left(A^{*}|B|^{2} A\right)+f\left(D\left|C^{*}\right|^{2} D^{*}\right)\right\|^{2}-\inf _{\|x\|=1} \phi(x)
$$

Proof. Let $y=x$ in (2.9), we get

$$
\begin{aligned}
f\left(|\langle D C B A x, x\rangle|^{2}\right) \leq 2^{-\frac{2}{r}} & \left(\left\langle f^{r}\left(A^{*}|B|^{2} A\right) x, x\right\rangle+\left\langle f^{r}\left(D\left|C^{*}\right|^{2} D^{*}\right) x, x\right\rangle\right)^{\frac{2}{r}} \\
& -\frac{1}{4}\left[\left\langle f\left(A^{*}|B|^{2} A\right) x, x\right\rangle-\left\langle f\left(D\left|C^{*}\right|^{2} D^{*}\right) x, x\right\rangle\right]
\end{aligned}
$$

Taking the supremum over all univt vector $x \in \mathscr{H}$, we get the required result. The particular case follows by setting $y=x$ in (2.10) and then taking the supremum over all univt vector $x \in \mathscr{H}$.

Corollary 3.12. Let $A, B, C, D \in \mathscr{B}(\mathscr{H})$. Then,

$$
w^{2 \lambda}(D C B A) \leq 2^{-\frac{2}{r}}\left\|\left(A^{*}|B|^{2} A\right)^{r \lambda}+\left(D\left|C^{*}\right|^{2} D^{*}\right)^{r \lambda}\right\|^{\frac{2}{r}}-\inf _{\|x\|=1} \phi(x)
$$

where,

$$
\phi_{1}(x):=\frac{1}{4}\left[\left\langle\left[\left(A^{*}|B|^{2} A\right)^{\lambda}-\left(D\left|C^{*}\right|^{2} D^{*}\right)^{\lambda}\right] x, x\right\rangle\right]
$$

In particular case, we have

$$
w^{2 \lambda}(D C B A) \leq \frac{1}{4}\left\|\left(A^{*}|B|^{2} A\right)^{\lambda}+\left(D\left|C^{*}\right|^{2} D^{*}\right)^{\lambda}\right\|^{2}-\inf _{\|x\|=1} \phi_{1}(x)
$$

Proof. Applying Theorem 3.11 for $f(t)=t^{\lambda}(\lambda \geq 1)$, we get the required result.

Corollary 3.13. Let $T \in \mathscr{B}(\mathscr{H})$. Let $f: \mathbb{R} \rightarrow(0, \infty)$ be a positive, increasing, convex and supermultiplicative i.e., $f(t s) \leq f(t) f(s)$ for all $t, s \in \mathbb{R}$.

$$
f\left(w^{2}\left(T|T|^{\alpha+\beta-1}\right)\right) \leq 2^{-\frac{2}{r}}\left\|f^{r}\left(|T|^{2 \alpha}\right)+f^{r}\left(\left|T^{*}\right|^{2 \beta}\right)\right\|^{\frac{2}{r}}-\inf _{\|x\|=1} \Psi(x)
$$

where,

$$
\Psi(x):=\frac{1}{4}\left[\left\langle\left[f\left(|T|^{2 \alpha}\right)-f\left(\left|T^{*}\right|^{2 \beta}\right)\right] x, x\right\rangle\right]
$$

Proof. Let $D=U, B=1_{\mathscr{H}}, C=|T|^{\beta}$ and $A=|T|^{\alpha}$ such that $\alpha+\beta \geq 1$ in (3.8).

Corollary 3.14. Let $T \in \mathscr{B}(\mathscr{H})$. Then,

$$
w^{2 \lambda}\left(T|T|^{\alpha+\beta-1}\right) \leq 2^{-\frac{2}{r}}\left\||T|^{2 r \alpha \lambda}+\left|T^{*}\right|^{2 r \beta \lambda}\right\|^{\frac{2}{r}}-\inf _{\|x\|=1} \Psi_{1}(x)
$$

for all $\alpha, \beta \geq 0$ such that $\alpha+\beta \geq 1$, where

$$
\Psi_{1}(x):=\frac{1}{4}\left\langle\left[|T|^{2 \alpha \lambda}-\left|T^{*}\right|^{2 \beta \lambda}\right] x, x\right\rangle
$$

Proof. Setting $f(t)=t^{\lambda}(\lambda \geq 1)$ in Corollary 3.13 we get the required result. 
Remark 3.15. By choosing $\alpha=\beta=\frac{1}{2}$ in (3.13), we get

$$
w^{2 \lambda}(T) \leq 2^{-\frac{2}{r}}\left\||T|^{r \lambda}+\left|T^{*}\right|^{r \lambda}\right\|^{\frac{2}{r}}-\frac{1}{4} \inf _{\|x\|=1}\left\langle\left[|T|^{\lambda}-\left|T^{*}\right|^{\lambda}\right] x, x\right\rangle
$$

for all $r, \lambda \geq 1$.

Also, for $r=1$ in (3.14) we get

$$
w^{2 \lambda}(T) \leq \frac{1}{4}\left\||T|^{\lambda}+\left|T^{*}\right|^{\lambda}\right\|^{2}-\frac{1}{4} \inf _{\|x\|=1}\left\langle\left[|T|^{\lambda}-\left|T^{*}\right|^{\lambda}\right] x, x\right\rangle
$$

for all $\lambda \geq 1$.

In general, for $\lambda=1$ in (3.14) we have

$$
w^{2}(T) \leq 2^{-\frac{2}{r}}\left\||T|^{r}+\left|T^{*}\right|^{r}\right\|^{\frac{2}{r}}-\frac{1}{4} \inf _{\|x\|=1}\left\langle\left[|T|-\left|T^{*}\right|\right] x, x\right\rangle
$$

for all $r \geq 1$.

Numerical radius inequality of special type of Hilbert space operators for commutators can be established as follows:

Lemma 3.16. Let $A_{1}, A_{2}, B_{1}, B_{2}, C_{1}, C_{2}, D_{1}, D_{2} \in \mathscr{B}(\mathscr{H})$. Then, for all $r \geq 1$ the inequality

$$
\begin{aligned}
& \left|\left\langle\left(D_{1} C_{1} B_{1} A_{1}+D_{2} C_{2} B_{2} A_{2}\right) x, y\right\rangle\right| \\
& \leq 2^{-\frac{1}{r}}\left(\left\langle\left(A_{1}^{*}\left|B_{1}\right|^{2} A_{1}\right)^{r} x, x\right\rangle+\left\langle\left(D_{1}\left|C_{1}^{*}\right|^{2} D_{1}^{*}\right)^{r} y, y\right\rangle\right)^{\frac{1}{r}} \\
& \quad-\frac{1}{2}\left(\left\langle A_{1}^{*}\left|B_{1}\right|^{2} A_{1} x, x\right\rangle^{\frac{1}{2}}-\left\langle D_{1}\left|C_{1}^{*}\right|^{2} D_{1}^{*} y, y\right\rangle^{\frac{1}{2}}\right)^{2} \\
& +2^{-\frac{1}{r}}\left(\left\langle\left(A_{2}^{*}\left|B_{2}\right|^{2} A_{2}\right)^{r} x, x\right\rangle+\left\langle\left(D_{2}\left|C_{2}^{*}\right|^{2} D_{2}^{*}\right)^{r} y, y\right\rangle\right)^{\frac{1}{r}} \\
& \quad-\frac{1}{2}\left(\left\langle A_{2}^{*}\left|B_{2}\right|^{2} A_{2} x, x\right\rangle^{\frac{1}{2}}-\left\langle D_{2}\left|C_{2}^{*}\right|^{2} D_{2}^{*} y, y\right\rangle^{\frac{1}{2}}\right)^{2}
\end{aligned}
$$

holds for all vectors $x, y \in \mathscr{H}$. 
Proof. Employing the triangle inequality and the inequality (1.6), we have

$$
\begin{aligned}
& \left|\left\langle\left(D_{1} C_{1} B_{1} A_{1}+D_{2} C_{2} B_{2} A_{2}\right) x, y\right\rangle\right| \\
& \leq\left|\left\langle\left(D_{1} C_{1} B_{1} A_{1}\right) x, y\right\rangle\right|+\left|\left\langle\left(D_{2} C_{2} B_{2} A_{2}\right) x, y\right\rangle\right| \\
& \leq\left\langle A_{1}^{*}\left|B_{1}\right|^{2} A_{1} x, x\right\rangle^{\frac{1}{2}}\left\langle D_{1}\left|C_{1}^{*}\right|^{2} D_{1}^{*} y, y\right\rangle^{\frac{1}{2}} \\
& +\left\langle A_{2}^{*}\left|B_{2}\right|^{2} A_{2} x, x\right\rangle^{\frac{1}{2}}\left\langle D_{2}\left|C_{2}^{*}\right|^{2} D_{2}^{*} y, y\right\rangle^{\frac{1}{2}} \\
& \leq 2^{-\frac{1}{r}}\left(\left\langle A_{1}^{*}\left|B_{1}\right|^{2} A_{1} x, x\right\rangle^{r}+\left\langle D_{1}\left|C_{1}^{*}\right|^{2} D_{1}^{*} y, y\right\rangle^{r}\right)^{\frac{1}{r}} \\
& -\frac{1}{2}\left(\left\langle A_{1}^{*}\left|B_{1}\right|^{2} A_{1} x, x\right\rangle^{\frac{1}{2}}-\left\langle D_{1}\left|C_{1}^{*}\right|^{2} D_{1}^{*} y, y\right\rangle^{\frac{1}{2}}\right)^{2} \\
& +2^{-\frac{1}{r}}\left(\left\langle A_{2}^{*}\left|B_{2}\right|^{2} A_{2} x, x\right\rangle^{r}+\left\langle D_{2}\left|C_{2}^{*}\right|^{2} D_{2}^{*} y, y\right\rangle^{r}\right)^{\frac{1}{r}} \\
& -\frac{1}{2}\left(\left\langle A_{2}^{*}\left|B_{2}\right|^{2} A_{2} x, x\right\rangle^{\frac{1}{2}}-\left\langle D_{2}\left|C_{2}^{*}\right|^{2} D_{2}^{*} y, y\right\rangle^{\frac{1}{2}}\right)^{2} \\
& \leq 2^{-\frac{1}{r}}\left(\left\langle\left(A_{1}^{*}\left|B_{1}\right|^{2} A_{1}\right)^{r} x, x\right\rangle+\left\langle\left(D_{1}\left|C_{1}^{*}\right|^{2} D_{1}^{*}\right)^{r} y, y\right\rangle\right)^{\frac{1}{r}} \\
& -\frac{1}{2}\left(\left\langle A_{1}^{*}\left|B_{1}\right|^{2} A_{1} x, x\right\rangle^{\frac{1}{2}}-\left\langle D_{1}\left|C_{1}^{*}\right|^{2} D_{1}^{*} y, y\right\rangle^{\frac{1}{2}}\right)^{2} \\
& +2^{-\frac{1}{r}}\left(\left\langle\left(A_{2}^{*}\left|B_{2}\right|^{2} A_{2}\right)^{r} x, x\right\rangle+\left\langle\left(D_{2}\left|C_{2}^{*}\right|^{2} D_{2}^{*}\right)^{r} y, y\right\rangle\right)^{\frac{1}{r}} \\
& -\frac{1}{2}\left(\left\langle A_{2}^{*}\left|B_{2}\right|^{2} A_{2} x, x\right\rangle^{\frac{1}{2}}-\left\langle D_{2}\left|C_{2}^{*}\right|^{2} D_{2}^{*} y, y\right\rangle^{\frac{1}{2}}\right)^{2}
\end{aligned}
$$

for all vectors $x, y \in \mathscr{H}$, which proves the result.

Corollary 3.17. Let $A_{1}, A_{2}, B_{1}, B_{2}, C_{1}, C_{2}, D_{1}, D_{2} \in \mathscr{B}(\mathscr{H})$. Then, the inequality

$$
\begin{aligned}
w( & \left.\left.D_{1} C_{1} B_{1} A_{1}+D_{2} C_{2} B_{2} A_{2}\right)\right) \\
\leq 2^{-\frac{1}{r}} & \left\|\left(A_{1}^{*}\left|B_{1}\right|^{2} A_{1}\right)^{r}+\left(D_{1}\left|C_{1}^{*}\right|^{2} D_{1}^{*}\right)^{r}\right\|^{\frac{1}{r}} \\
& +2^{-\frac{1}{r}}\left\|\left(A_{2}^{*}\left|B_{2}\right|^{2} A_{2}\right)^{r}+\left(D_{2}\left|C_{2}^{*}\right|^{2} D_{2}^{*}\right)^{r}\right\|^{\frac{1}{r}} \\
& -\frac{1}{2} \inf _{\|x\|=1}\left(\left\langle A_{1}^{*}\left|B_{1}\right|^{2} A_{1} x, x\right\rangle^{\frac{1}{2}}-\left\langle D_{1}\left|C_{1}^{*}\right|^{2} D_{1}^{*} x, x\right\rangle^{\frac{1}{2}}\right)^{2} \\
& -\frac{1}{2} \inf _{\|x\|=1}\left(\left\langle A_{2}^{*}\left|B_{2}\right|^{2} A_{2} x, x\right\rangle^{\frac{1}{2}}-\left\langle D_{2}\left|C_{2}^{*}\right|^{2} D_{2}^{*} x, x\right\rangle^{\frac{1}{2}}\right)^{2}
\end{aligned}
$$

holds for all $r \geq 1$.

Proof. Let $y=x$ in (3.15) and then taking the supremum over all univt vector $x \in \mathscr{H}$, we get the required result. 
Corollary 3.18. Let $A, B, C, D \in \mathscr{B}(\mathscr{H})$. Then

$$
\begin{aligned}
& w\left(\left(D_{1} C_{1} B_{1} A_{1}+D_{2} C_{2} B_{2} A_{2}\right)\right) \\
& \leq \frac{1}{2}\left\|A_{1}^{*}\left|B_{1}\right|^{2} A_{1}+D_{1}\left|C_{1}^{*}\right|^{2} D_{1}^{*}+A_{2}^{*}\left|B_{2}\right|^{2} A_{2}+D_{2}\left|C_{2}^{*}\right|^{2} D_{2}^{*}\right\| \\
& \quad-\frac{1}{2} \inf _{\|x\|=1}\left(\left\langle A_{1}^{*}\left|B_{1}\right|^{2} A_{1} x, x\right\rangle^{\frac{1}{2}}-\left\langle D_{1}\left|C_{1}^{*}\right|^{2} D_{1}^{*} x, x\right\rangle^{\frac{1}{2}}\right)^{2} \\
& \quad-\frac{1}{2} \inf _{\|x\|=1}\left(\left\langle A_{2}^{*}\left|B_{2}\right|^{2} A_{2} x, x\right\rangle^{\frac{1}{2}}-\left\langle D_{2}\left|C_{2}^{*}\right|^{2} D_{2}^{*} x, x\right\rangle^{\frac{1}{2}}\right)^{2}
\end{aligned}
$$

for all vectors $x, y \in \mathscr{H}$.

Proof. Let $y=x$ in (3.15) and consider $r=1$. In the proof of (3.15) combining the inner products then taking the supremum over all univt vector $x \in \mathscr{H}$, we get the required result.

In special cases, a particular choice of $A, B, C, D$ in the Corollaries 3.17 and 3.18 would give the same results proved recently, by Alomari in [4], as follows:

Corollary 3.19. Let $T, S \in(B)(\mathscr{H}), \alpha, \beta, \gamma, \delta \geq 0$ such that $\alpha+\beta \geq 1$ and $\gamma+\delta \geq 1$. Then

$$
\begin{aligned}
& w\left(T|T|^{\alpha+\beta-1}+S|S|^{\gamma+\delta-1}\right) \\
& \leq 2^{-\frac{1}{r}}\left\||T|^{2 r \alpha}+\left|T^{*}\right|^{2 r \beta}\right\|^{\frac{1}{r}}+2^{-\frac{1}{r}}\left\||S|^{2 r \gamma}+\left|S^{*}\right|^{2 r \delta}\right\|^{\frac{1}{r}} \\
& \quad-\frac{1}{2} \inf _{\|x\|=1}\left(\left\langle|T|^{2 \alpha} x, x\right\rangle^{\frac{1}{2}}-\left\langle\left|T^{*}\right|^{2 \beta} x, x\right\rangle^{\frac{1}{2}}\right)^{2} \\
& \quad-\frac{1}{2} \inf _{\|x\|=1}\left(\left\langle|S|^{2 \gamma} x, x\right\rangle^{\frac{1}{2}}-\left\langle\left|S^{*}\right|^{2 \delta} x, x\right\rangle^{\frac{1}{2}}\right)^{2}
\end{aligned}
$$

for all $r \geq 1$.

Proof. Let $D=U, B=1_{\mathscr{H}}, C=|T|^{\beta}$ and $A=|T|^{\alpha}$ such that $\alpha+\beta \geq 1$ in (3.16), then we have

$$
D C B A=U|T|^{\beta}|T|^{\alpha}=U|T||T|^{\alpha+\beta-1}=T|T|^{\alpha+\beta-1},
$$

also, we have $A^{*}|B|^{2} A=|T|^{2 \alpha}$ and $D\left|C^{*}\right|^{2} D^{*}=U|T|^{2 \beta} U^{*}=|T|^{2 \beta}$

Corollary 3.20. Let $T, S \in(B)(\mathscr{H}), \alpha, \beta, \gamma, \delta \geq 0$ such that $\alpha+\beta \geq 1$ and $\gamma+\delta \geq 1$. Then

$$
\begin{aligned}
w\left(T|T|^{\alpha+\beta-1}+S|S|^{\gamma+\delta-1}\right) \leq \frac{1}{2} & \left\||T|^{2 \alpha}+\left|T^{*}\right|^{2 \beta}+|S|^{2 \gamma}+\left|S^{*}\right|^{2 \delta}\right\| \\
& -\frac{1}{2} \inf _{\|x\|=1}\left(\left\langle|T|^{2 \alpha} x, x\right\rangle^{\frac{1}{2}}-\left\langle\left|T^{*}\right|^{2 \beta} x, x\right\rangle^{\frac{1}{2}}\right)^{2} \\
& -\frac{1}{2} \inf _{\|x\|=1}\left(\left\langle|S|^{2 \gamma} x, x\right\rangle^{\frac{1}{2}}-\left\langle\left|S^{*}\right|^{2 \delta} x, x\right\rangle^{\frac{1}{2}}\right)^{2} .
\end{aligned}
$$


Proof. Let $D=U, B=1_{\mathscr{H}}, C=|T|^{\beta}$ and $A=|T|^{\alpha}$ such that $\alpha+\beta \geq 1$ in (3.17).

Remark 3.21. Setting $\alpha=\beta=\gamma=\delta=\frac{1}{2}$ in (3.19), we get

$$
\begin{gathered}
w(T+S) \leq \frac{1}{2}\left\||T|+\left|T^{*}\right|+|S|+\left|S^{*}\right|\right\|-\frac{1}{2} \inf _{\|x\|=1}\left(\langle|T| x, x\rangle^{\frac{1}{2}}-\left\langle\left|T^{*}\right| x, x\right\rangle^{\frac{1}{2}}\right)^{2} \\
-\frac{1}{2} \inf _{\|x\|=1}\left(\langle|S| x, x\rangle^{\frac{1}{2}}-\left\langle\left|S^{*}\right| x, x\right\rangle^{\frac{1}{2}}\right)^{2}
\end{gathered}
$$

In particular, take $S=T$ we get

$$
w(T) \leq \frac{1}{2}\left\||T|+\left|T^{*}\right|+\right\|-\frac{1}{2} \inf _{\|x\|=1}\left(\langle|T| x, x\rangle^{\frac{1}{2}}-\left\langle\left|T^{*}\right| x, x\right\rangle^{\frac{1}{2}}\right)^{2}
$$

Remark 3.22. Setting $\alpha=\beta=\gamma=\delta=1$ in (3.19), we get

$$
\begin{aligned}
w(T|T|+S|S|) \leq \frac{1}{2} & \left\||T|^{2}+\left|T^{*}\right|^{2}+|S|^{2}+\left|S^{*}\right|^{2}\right\| \\
& -\frac{1}{2} \inf _{\|x\|=1}\left(\left\langle|T|^{2} x, x\right\rangle^{\frac{1}{2}}-\left\langle\left|T^{*}\right|^{2} x, x\right\rangle^{\frac{1}{2}}\right)^{2} \\
& -\frac{1}{2} \inf _{\|x\|=1}\left(\left\langle|S|^{2} x, x\right\rangle^{\frac{1}{2}}-\left\langle\left|S^{*}\right|^{2} x, x\right\rangle^{\frac{1}{2}}\right)^{2}
\end{aligned}
$$

In particular, take $S=T$, we get

$$
\begin{aligned}
w(T|T|) & \leq \frac{1}{2}\left\||T|^{2}+\left|T^{*}\right|^{2}\right\|-\frac{1}{2} \inf _{\|x\|=1}\left(\left\langle|T|^{2} x, x\right\rangle^{\frac{1}{2}}-\left\langle\left|T^{*}\right|^{2} x, x\right\rangle^{\frac{1}{2}}\right)^{2} \\
& =\frac{1}{2}\left\|T^{*} T+T T^{*}\right\|-\frac{1}{2} \inf _{\|x\|=1}\left(\left\langle|T|^{2} x, x\right\rangle^{\frac{1}{2}}-\left\langle\left|T^{*}\right|^{2} x, x\right\rangle^{\frac{1}{2}}\right)^{2}
\end{aligned}
$$

\section{REFERENCES}

1. Y. Al-Manasrah and F. Kittaneh, A generalization of two refined Young inequalities, Positivity, 19 (2015), 757-768.

2. M.W. Alomari, On the generalized mixed Schwarz inequality, Proceedings of the Institute of Mathematics and Mechanics, National Academy of Sciences of Azerbaijan, to appear

3. M.W. Alomari, Refinements of some numerical radius inequalities for Hilbert space operators, Linear and Multilinear Algebra, (2019), DOI: 10.1080/03081087.2019.1624682

4. M.W. Alomari, Improvements of some numerical radius inequalities, ArXiv, (2019).

5. S.S. Dragomir, Inequalities for the numerical radius of linear operators in Hilbert spaces, SpringerBriefs in Mathematics, 2013.

6. S.S. Dragomir, Some inequalities for the norm and the numerical radius of linear operator in Hilbert spaces, Tamkang J. Math., 39 (1) (2008), 1-7.

7. S.S. Dragomir, Some Inequalities generalizing Kato's and Furuta's results, FILOMAT, 28 (1) (2014), 179-195.

8. T. Furuta, An extension of the Heinz-Kato theorem, Proc. Amer. Math. Soc., 120 (1994), no. $3,785-787$.

9. P.R. Halmos, A Hilbert space problem book, Van Nostrand Company, Inc., Princeton, N.J., 1967.

10. H.R. Moradi, S. Furuichi, F.C. Mitroi and R. Naseri, An extension of Jensen's operator inequality and its application to Young inequality, Rev. R. Acad. Cienc. Exactas Fs. Nat. Ser. A Mat., 113 (2) (2019), 605-614. 
11. F. Kittaneh, M.S. Moslehian and T. Yamazaki, Cartesian decomposition and numerical radius inequalities, Linear Algebra Appl. 471 (2015), 46-53.

12. F. Kittaneh, Numerical radius inequalities for Hilbert space operators, Studia Math., 168 (1) (2005), 73-80.

13. F. Kittaneh and Y. Manasrah, Improved Young and Heinz inequalities for matrices, $J$. Math. Anal. Appl. 361 (2010), 262-269.

14. F. Kittaneh, A numerical radius inequality and an estimate for the numerical radius of the Frobenius companion matrix, Studia Math., 158 (2003), 11-17.

15. F. Kittaneh, Notes on some inequalities for Hilbert Space operators, Publ. Res. Inst. Math. Sci., 24 (2) (1988), 283-293.

16. T. Kato, Notes on some inequalities for linear operators, Math. Ann., 125 (1952), 208-212.

17. C.-S. Lin and S.S. Dragomir, On High-power Operator inequalities and spectral radii of operators, Publ. RIMS, Kyoto Univ., 42 (2006), 391-397.

18. J. Pečarić, T. Furuta, J.M. Hot and Y. Seo, Mond-Pečarić method in operator inequalities, inequalities for bounded selfadjoint operators on a Hilbert space, Monographs in Inequalities, Element, Zagreb, 2005.

19. W. Reid, Symmetrizable completely continuous linear tarnsformations in Hilbert space, Duke Math., 18 (1951), 41-56.

20. M. Sattari, M.S. Moslehian and T. Yamazaki, Some genaralized numerical radius inequalities for Hilbert space operators, Linear Algebra Appl., 470 (2014), 1-12.

21. A. Sheikhhosseini, M. S. Moslehian and K. Shebrawi, Inequalities for generalized Euclidean operator radius via Young's inequality, J. Math. Anal. Appl. 445 (2017), 1516-1529.

22. T. Yamazaki, On upper and lower bounds of the numerical radius and an equality condition, Studia Math., 178 (2007), 83-89. 